\title{
Spectroscopic studies on the interaction of efonidipine with bovine serum albumin
}

\author{
N. Wang, L. Ye, B.Q. Zhao and J.X. Yu \\ School of Chemical Biology and Pharmaceutical Sciences, Capital Medical University, \\ Beijing, P.R. China \\ Correspondence to: L. Ye, School of Chemical Biology and Pharmaceutical Sciences, Capital Medical \\ University, Beijing 100069, P.R. China \\ E-mail: lingye@ccmu.edu.cn
}

\begin{abstract}
Efonidipine hydrochloride is an antihypertensive and antianginal agent with fewer side effects and is better tolerated in the treatment of hypertension with renal impairment. Its interaction with bovine serum albumin (BSA) is of great use for the understanding of the pharmacokinetic and pharmacodynamic mechanisms of the drug. The binding of efonidipine to BSA was investigated by fluorescence spectroscopy and circular dichroism. BSA fluorescence was quenched by efonidipine, due to the fact that efonidipine quenched the fluorescence of tryptophan residues mainly by the collision mode. The thermodynamic parameters $\Delta H^{0}$ and $\Delta S^{0}$ were $68.04 \mathrm{~kJ} / \mathrm{mol}$ and $319.42 \mathrm{~J} \cdot \mathrm{mol}^{-1} \cdot \mathrm{K}^{-1}$, respectively, indicating that the hydrophobic interactions played a major role. The results of circular dichroism and synchronous fluorescence measurements showed that the binding of efonidipine to BSA led to a conformational change of BSA. The fraction of occupied sites $(\theta)$ for the 8-anilino-1-naphthaleinsulfonic acid (ANS)-BSA system is $85 \%$, whereas for the NZ-105-BSA system, it is $53 \%$, which suggests that the interaction of ANS with BSA is stronger than that of NZ-105 with BSA. Binding studies in the presence of ANS indicated that efonidipine competed with ANS for hydrophobic sites of BSA. The effects of metal ions on the binding constant of the efonidipine-BSA complex were also investigated. The presence of metal ions $\mathrm{Zn}^{2+}, \mathrm{Mg}^{2+}, \mathrm{Al}^{3+}, \mathrm{K}^{+}$, and $\mathrm{Ca}^{2+}$ increased the binding constant of efonidipine-BSA complex, which may prolong the storage period of NZ-105 in blood plasma and enhance its maximum effects.
\end{abstract}

Key words: Efonidipine; Bovine serum albumin; Fluorescence quenching; Synchronous fluorescence; Circular dichroism

Research supported by Beijing Natural Science Foundation of China (\#5073043), Scholastic Natural Science Foundation of Capital Medical University (\#2005ZR06), and Beijing Municipal Project for Developing Advanced Human Resources for Higher Education.

Received November 8, 2007. Accepted June 18, 2008

\section{Introduction}

It is known that drug-protein interactions greatly influence the absorption, distribution, metabolism, and excretion properties of drugs (1). Serum albumin is the most abundant protein in the circulatory system of a wide variety of organisms and plays an important role in the transport and deposition of many drugs (2-4). Thus, an understanding of the features of drug interactions with albumin can provide insights into drug therapy and design. Consequently, attention has been focused on the binding of drugs to albumin. Bovine serum albumin (BSA) is usually employed as a model protein because of its low cost and availability and because it is structurally homologous with human serum albumin (5).

Efonidipine hydrochloride (efonidipine, NZ-105; see structure in Figure 1) is a recently synthesized 1,4-dihydropyridine derivative that is used as an antihypertensive and antianginal agent. It has a potent vasodilating effect on vascular smooth muscle and a long-lasting hypotensive effect with a very slow onset (6). NZ-105 also has a chronotropic effect which may suppress tachycardia $(7,8)$. It has few side effects and is well tolerated for the treatment of hypertension in the presence of renal impairment (9). Thus, NZ-105 is a member of a new generation of drugs for the treatment of hypertensive patients with chronic renal disease. However, detailed information on the binding of NZ-105 to BSA is not available. 
Spectral methods can reveal the binding of drugs with albumin at low concentrations. The fluorescence quenching technique is often used to monitor the molecular interactions because of its high sensitivity, reproducibility and relatively easy use $(10,11)$. In the present study, we investigated the interactions of NZ-105 with BSA as well as the effect of common metal ions on these interactions by fluorescence spectroscopy. Additionally, binding of NZ105 to BSA in the presence of the fluorescence probe 8anilino-1-naphthalein-sulfonic acid (ANS) was also determined. The effects of NZ-105 on the conformational changes of BSA were investigated by synchronous fluorescence spectroscopy and circular dichroism (CD).

\section{Material and Methods}

\section{Chemicals}

BSA (>96\%, essentially fatty acid free, lyophilized powder) and ANS were purchased from Sigma (USA). Efonidipine was obtained from Garlin Pharmaceutical Co. (China). All other chemicals $\left(\mathrm{KCl}, \mathrm{MgCl}_{2} \cdot 6 \mathrm{H}_{2} \mathrm{O}, \mathrm{CaCl}_{2}\right.$, $\mathrm{ZnCl} 2, \mathrm{AlCl}_{3} \cdot 6 \mathrm{H}_{2} \mathrm{O}$ ) were of analytical grade and used without further purification.

\section{Instruments}

Fluorescence and synchronous measurements were performed using a 1-cm quartz cell on an RF-5301PC spectrofluorophotometer (Shimadzu, Japan) equipped with an SB-11 water bath (Eyela). The absorption spectrum was obtained with a UV-2250 UV-Vis spectrophotometer (Shimadzu). The CD measurements were recorded on a JASCO-J-810 spectropolarimeter (JASCO, Japan) using a $0.1-\mathrm{cm}$ quartz cell.

\section{Efonidipine-BSA interactions}

A 2.5-mL amount of $10 \mu \mathrm{M}$ BSA (based on a molecular mass of $68 \mathrm{kDa}$ ) was added to the quartz cell, NZ-105 was

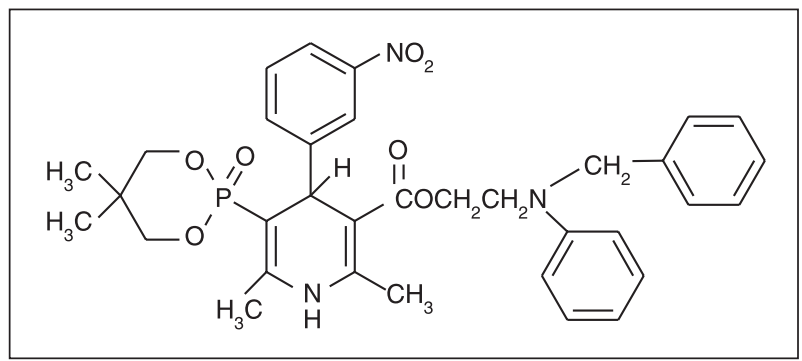

Figure 1. Structure of efonidipine (( \pm )-5-(5,5-dimethyl-1,3,2dioxaphosphorinan-2-yl)-2,6-dimethyl-4-(3-nitrophenyl)-1,4-dihydropyridine-3-carboxylic acid 2-(N-benzyl-N-phenylamino) ethyl ester $\mathrm{P}$-oxidehydrochloride ethanol). then gradually added to the cell and the NZ-105 concentrations ranged from 5 to $50 \mu \mathrm{M}$. Fluorescence emission spectra were recorded at three temperatures, i.e., 298, 304 , and $310 \mathrm{~K}$ upon excitation at $295 \mathrm{~nm}$. The excitation and emission slit widths were $3 \mathrm{~nm}$ each.

\section{Synchronous fluorescence measurements}

Synchronous fluorescence spectra of BSA with various concentrations of NZ-105 were obtained from 300 to $400 \mathrm{~nm}$ when $\Delta \lambda=60 \mathrm{~nm}$ and from 280 to $350 \mathrm{~nm}$ when $\Delta \lambda=15 \mathrm{~nm}$. The excitation and emission slit widths were 3 and $1.5 \mathrm{~nm}$, respectively.

\section{Circular dichroism measurements}

CD spectra of BSA in the absence and presence of NZ105 were recorded from 200 to $280 \mathrm{~nm}$ at $0.2-\mathrm{nm}$ intervals with five scans averaged for each spectrum. The overall concentration of BSA was kept at $1 \mu \mathrm{M}$, while the molar ratios of NZ-105 to BSA ranged from $0: 1$ to $3: 1$ and 10:1.

\section{Binding studies in the presence of ANS}

In the first set of experiments, the binding of NZ-105 or ANS to BSA was studied under the same experimental conditions. The final BSA concentration was $10 \mu \mathrm{M}$ and the concentration of NZ-105 or ANS varied from 2.5 to $25 \mu \mathrm{M}$. Fluorescence spectra of BSA were recorded from 300 to $500 \mathrm{~nm}$ with excitation at $295 \mathrm{~nm}$. In the second set of experiments, the binding sites of NZ-105 and ANS to BSA were determined simultaneously. BSA and ANS concentrations were fixed at 10 and $20 \mu \mathrm{M}$, respectively, and the concentration of NZ-105 varied from 0 to $20 \mu \mathrm{M}$. The fluorescence spectra of ANS were recorded from 390 to $550 \mathrm{~nm}$ with excitation at $370 \mathrm{~nm}$.

Effects of some common ions

The fluorescence spectra of NZ-105-BSA were recorded in the presence of metal ions $\left(\mathrm{Zn}^{2+}, \mathrm{Mg}^{2+}, \mathrm{Al}^{3+}, \mathrm{K}^{+}\right.$, and $\mathrm{Ca}^{2+}$ ) from 300 to $500 \mathrm{~nm}$ upon excitation at $295 \mathrm{~nm}$. Both BSA and metal ion concentrations were $10 \mu \mathrm{M}$.

\section{Results and Discussion}

\section{Fluorescence studies}

The intrinsic fluorescence of BSA when excited at 295 $\mathrm{nm}$ is mainly due to the presence of the two tryptophan residues: Trp-134 and Trp-212. Trp-212 is located within a hydrophobic binding pocket in the IIA sub-domain of the protein, whereas Trp-134 is located on the surface of the albumin molecule and more exposed to the environment (12). Though the tyrosine residue can also contribute to fluorescence, it presents very weak emission when ex- 
cited at $295 \mathrm{~nm}$.

The fluorescence spectra of BSA were recorded in the presence of increasing amount of NZ-105. As shown in Figure 2, the fluorescence intensity of BSA decreased regularly with increasing concentration of NZ-105, while the emission maximum and shape of the peaks remained almost unchanged. This indicated that NZ-105 could bind to BSA without altering the environment in the vicinity of the chromophore tryptophan residues.

The binding parameters were then obtained according to the following equation:

$\log \left(\frac{F_{0}-F}{F}\right)=\log K_{A}+n \log [Q]$

(Equation 1)

where $n$ is the number of binding sites, $K_{A}$ is the binding constant or the apparent association constant for drugprotein interaction. Values of $n$ and $K_{A}$ can thereby be determined from the intercept and slope by plotting $\log \left(F_{0}\right.$ - $F)$ / $F$ versus $\log [Q]$. The data in Table 1 show that the value of $n$ is approximately equal to 1 , indicating that there is one class of binding sites for NZ-105 in BSA (13). The value of the association constant increased from 5.58 to $16.14 \times 10^{4} \mathrm{M}^{-1}$ with increasing temperature from 298 to $310 \mathrm{~K}$. The increase in $K_{A}$ may be caused by a slight expansion of the binding site which might accommodate more NZ-105 molecules. The expansion of the binding site may also provide a larger hydrophobic area for the binding, thus leading to the increase in the value of $K_{A}$.

\section{Stern-Volmer analysis}

Fluorescence quenching is usually classified as dynamic quenching and static quenching (14). Dynamic quenching, or collisional quenching, results from collision between fluorophore and quencher. Static quenching is due to ground-state complex formation between fluorophore and quencher (15). To determine the mechanism of binding between BSA and NZ-105, fluorescence intensity data were analyzed by the Stern-Volmer equation (16):

$$
\frac{F_{0}}{F}=1+K_{s V}[Q]
$$

(Equation 2)

where $F$ and $F_{0}$ are the fluorescence intensity with and without the quencher (drug), $K_{s v}$ the Stern-Volmer quenching constant, and $[Q]$ the concentration of the drug. If the resulting plots exhibit a good linear relationship, this is generally indicative of the purely collisional quenching process or static quenching process (17).

However, the Stern-Volmer plot showed a minor positive deviation (Figure 3 ) indicating the presence of both static and dynamic quenching. Therefore, the data were processed based on a modified Stern-Volmer equation:
$\frac{F_{0}}{F}=1+K_{s V}[Q] \exp (V[Q]$

(Equation 3)

where $V$ is the static quenching constant, whose value can be obtained from Equation 3 by plotting $\frac{F_{o}-F}{F \exp (V[Q]}$ versus [Q] by varying $V$ until a linear plot is acquired (Figure 3 ). The $K_{s V}$ can then be obtained from the slope. The values of $V$ and $K_{s v}$ at different temperatures $(298,304$, and $310 \mathrm{~K})$ are presented in Table 2. As can be seen from Table 2, the values of $K_{s v}$ are much higher than the value of $V$, suggesting that the overall quenching is dominated by dynamic quenching, though a small static quenching component contributes to the positive deviation of the Stern-Volmer plot (18). This small part of static quenching can be explained by the fact that a minor fraction of the quencher molecule is very close to, or in contact with the fluorescent molecule at the exact moment it happens to be excited. The mechanism of efonidipine binding to BSA is similar to that of azelnidipine binding to BSA (19) due to the very similar structures of the two drugs.

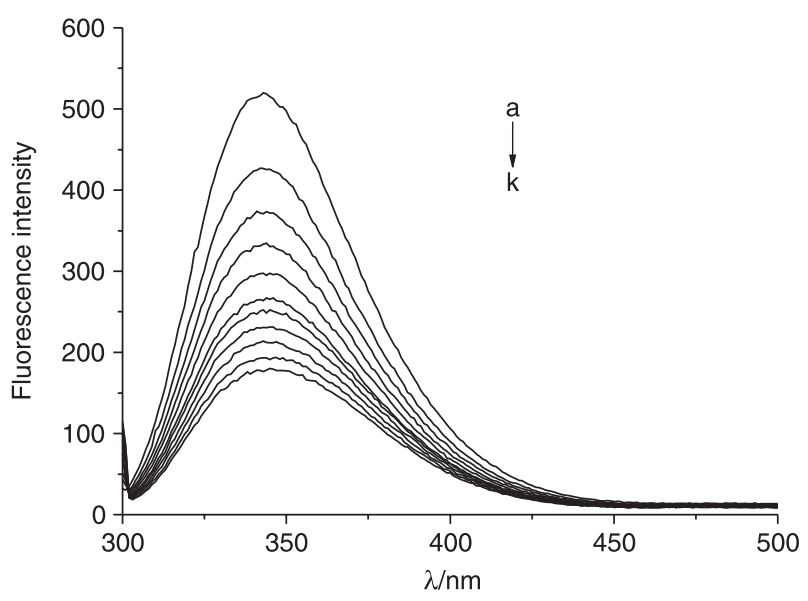

Figure 2. Fluorescence quenching spectra of BSA at different concentrations of efonidipine at $298 \mathrm{~K} ; C_{\mathrm{BSA}}=10 \mu \mathrm{M} ; C_{\text {efonidipine }}$ from a to k: 0 (a), 5 (b), 10 (c), 15 (d), 20 (e), 25 (f), 30 (g), 35 (h), 40 (i), $45(\mathrm{j})$, and $50(\mathrm{k}) \mu \mathrm{M}$.

Table 1. Binding parameters obtained from the interaction of efonidipine with bovine serum albumin.

\begin{tabular}{lcccc}
\hline $\mathrm{T}(\mathrm{K})$ & $K_{A}\left(\times 10^{4} \mathrm{M}^{-1}\right)$ & $n$ & $\mathrm{R}^{2}$ & $\mathrm{SD}$ \\
\hline 298 & 5.58 & 1.02 & 0.9997 & 0.0057 \\
304 & 10.38 & 1.08 & 0.9992 & 0.0104 \\
310 & 16.14 & 1.14 & 0.9995 & 0.0090
\end{tabular}

The parameters were calculated from the data in Figure 2 using Equation 1. $\mathrm{T}=$ temperature. 


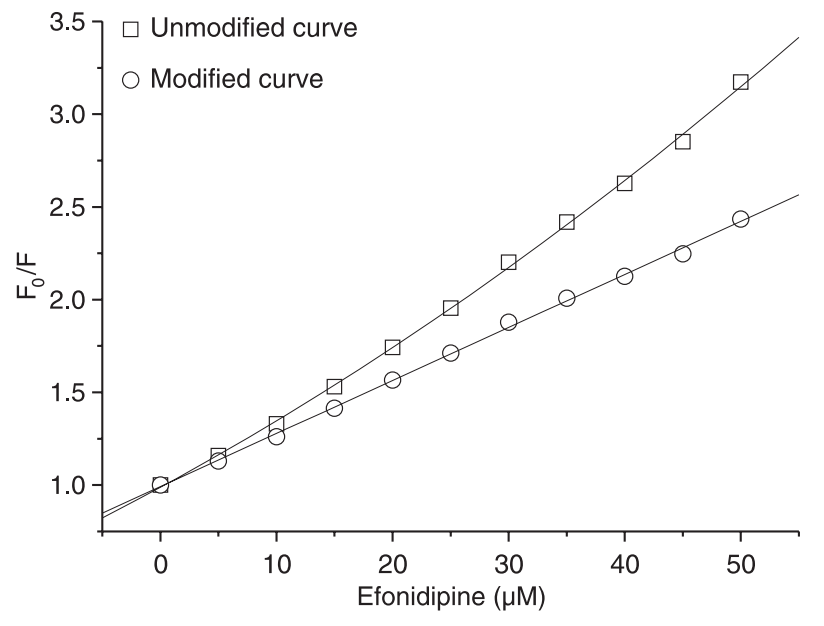

Figure 3. Modified and unmodified Stern-Volmer plots of the interaction of efonidipine with BSA. Original data are given in Figure 2.

Table 2. Quenching constants at three different temperatures for the efonidipine-bovine serum albumin system.

\begin{tabular}{ccccc}
\hline $\mathrm{T}(\mathrm{K})$ & $K_{S V}\left(\times 10^{4} \mathrm{M}^{-1}\right)$ & $V\left(\times 10^{3} \mathrm{M}^{-1}\right)$ & $\mathrm{R}^{2}$ & $\mathrm{SD}$ \\
\hline 298 & 3.45 & 4.00 & 0.9989 & 0.0264 \\
304 & 3.24 & 5.20 & 0.9997 & 0.0093 \\
310 & 2.84 & 5.30 & 0.9987 & 0.0177 \\
\hline
\end{tabular}

Equation 3 was used to calculate the data given in this table from those in Figure 3. $\mathrm{T}=$ temperature.

Table 3. Thermodynamic parameters of the efonidipine-bovine serum albumin interaction.

\begin{tabular}{ccccc}
\hline $\mathrm{T}(\mathrm{K})$ & $\begin{array}{c}K_{A} \\
\left(\times 10^{4} \mathrm{M}^{-1}\right)\end{array}$ & $\begin{array}{c}\Delta G^{0} \\
(\mathrm{~kJ} / \mathrm{mol})\end{array}$ & $\begin{array}{c}\Delta H^{0} \\
(\mathrm{~kJ} / \mathrm{mol})\end{array}$ & $\begin{array}{c}\Delta S^{0} \\
\left(\mathrm{~J} \cdot \mathrm{mol}^{-1} \cdot \mathrm{K}^{-1}\right)\end{array}$ \\
\hline 298 & 5.58 & -27.08 & & \\
304 & 10.38 & -29.19 & 68.04 & 319.42 \\
310 & 16.14 & -30.91 & & \\
\hline
\end{tabular}

Data were calculated with Equation $5 . \mathrm{T}=$ temperature.

Type of interaction force between efonidipine and BSA

The interaction forces between drugs and biomolecules include hydrophobic force, electrostatic interactions, van der Waals interactions, hydrogen bonds, etc. (20). In order to identify the interaction force of NZ-105 with BSA, the thermodynamic parameters, i.e., free energy changes $\left(\Delta G^{0}\right)$, enthalpy changes $\left(\Delta H^{0}\right)$, and entropy changes $\left(\Delta S^{0}\right)$ of the interactions were calculated from the following equations: $\ln K=-\frac{\Delta H^{o}}{R T}+\frac{\Delta S^{o}}{R}$

(Equation 4)

$\Delta G^{0}=-R T \ln K=\Delta H^{0}-T \Delta S^{0}$

(Equation 5)

where, $K$ corresponds to the binding constant $K_{A}$ and $R$ is the gas constant. The thermodynamic parameters are summarized in Table 3. The negative value of $\Delta G^{0}$ identifies NZ-105 binding to BSA as a spontaneous process. The positive $\Delta H^{0}$ and $\Delta S^{0}$ values indicate that the force acting between NZ-105 and BSA is mainly a hydrophobic interaction (21). Thus, the non-polar hydrophobic groups of serum albumins may be responsible for the main effect determining the binding of NZ-105 and serum albumin. This, together with the number of binding sites, suggest that the binding site for NZ-105 on BSA is primarily on the Trp-212 residue, which is located within a hydrophobic binding pocket of the protein.

\section{Conformational investigations}

Synchronous fluorescence spectra (Figure 4) of BSA with different amounts of NZ-105 were measured to explore the conformational change of BSA upon the addition of NZ-105. The synchronous fluorescence spectra provide information about the molecular environment in the vicinity of the chromosphere molecules and the changes of maximum emission wavelength reflect the conformational changes of BSA (22). When the $\Delta \lambda$ between excitation wavelength and emission wavelength was fixed at 15 and $60 \mathrm{~nm}$, the synchronous fluorescence spectra gave the characteristic information of tyrosine and tryptophan residues, respectively (23).

The quenching of the fluorescence intensity of tryptophan residues is stronger than that of tyrosine residues, indicating that tryptophan residues contribute greatly to the intrinsic fluorescence of BSA. It is also noted that there was a red shift of tryptophan residue fluorescence emission maximum upon the addition of NZ-105, whereas the emission maximum of tyrosine remained unchanged. The red shift indicates that the conformation of BSA is changed and the hydrophobicity decreases. The decreased hydrophobicity may be attributed to the binding of NZ-105 to $B S A$, which causes the extension of peptide strand of the BSA.

To confirm that there is a conformational change of BSA upon the addition of NZ-105, the CD spectra of BSA with and without NZ-105 were measured. As shown in Figure 5 , the $C D$ spectra of BSA exhibit two negative bands in the UV region at 209 and $222 \mathrm{~nm}$, characteristic of the $\alpha$-helical structure of the protein (24). The binding of 
NZ-105 to BSA reduced the intensity of both bands, indicating a decrease of the $\alpha$-helical content in the protein. That is, NZ-105 binds to the amino acid residue of the polypeptide chain of BSA and destroys their hydrogen bonding networks (25). Nevertheless, the CD spectra of BSA in the presence and absence of NZ-105 are similar in shape, implying that the structure of BSA after the binding of NZ-105 continues to be predominantly $\alpha$-helical.

\section{Energy transfer from BSA to efonidipine}

According to Föster's theory, the energy transfer effect is related not only to the distance between the acceptor and donor $\left(r_{0}\right)$, but also to the critical energy transfer distance $\left(R_{0}\right)$ :

$E=1-\frac{F}{F_{o}}=\frac{R_{o}^{6}}{R_{o}^{6}+r^{6}}$

(Equation 6)

where $E$ is the energy transfer efficiency and $R_{0}$ is the critical distance when the transfer efficiency is $50 \%$ and can be calculated from the following equation:

$R_{o}^{6}=8.8 \times 10^{-25} k^{2} N^{-4} \Phi J$

(Equation 7)

In Equation 7, $k^{2}$ is the spatial orientation factor of the dipole, $N$ is the refractive index of the medium, and $\phi$ is the fluorescence quantum yield of the donor. $J$ is the effect of the spectral overlap between the emission spectrum of the donor and the absorption spectrum of the acceptor (Figure 6). Therefore,

$J=\frac{\sum F(\lambda) \varepsilon(\lambda) \lambda^{4} \Delta \lambda}{\sum F(\lambda) \Delta \lambda}$

(Equation 8)

where $F(\lambda)$ is the fluorescence intensity of the fluorescent donor at wavelength $\lambda$, and $\varepsilon(\lambda)$ is the molar absorptivity of the acceptor at wavelength $\lambda$.

Under the present experimental conditions, $k=2 / 3, n=$ 1.336, $\phi=0.15$ (26). According to Equations 6 to 8, we can calculate that $J=2.32 \times 10^{-15} \mathrm{~cm}^{3} \mathrm{~L} \mathrm{~mol}^{-1}, R_{0}=2.00 \mathrm{~nm}, E$ $=0.301$, and $r=2.30 \mathrm{~nm}$. Since the distance between donor and acceptor for the NZ-105-BSA complex is in the 2- to 8-nm scale, non-radiative energy transfer occurs between NZ-105 and BSA with high probability.

\section{Binding studies in the presence of ANS}

When two different kinds of drugs are administered simultaneously, it is possible that one will compete with the
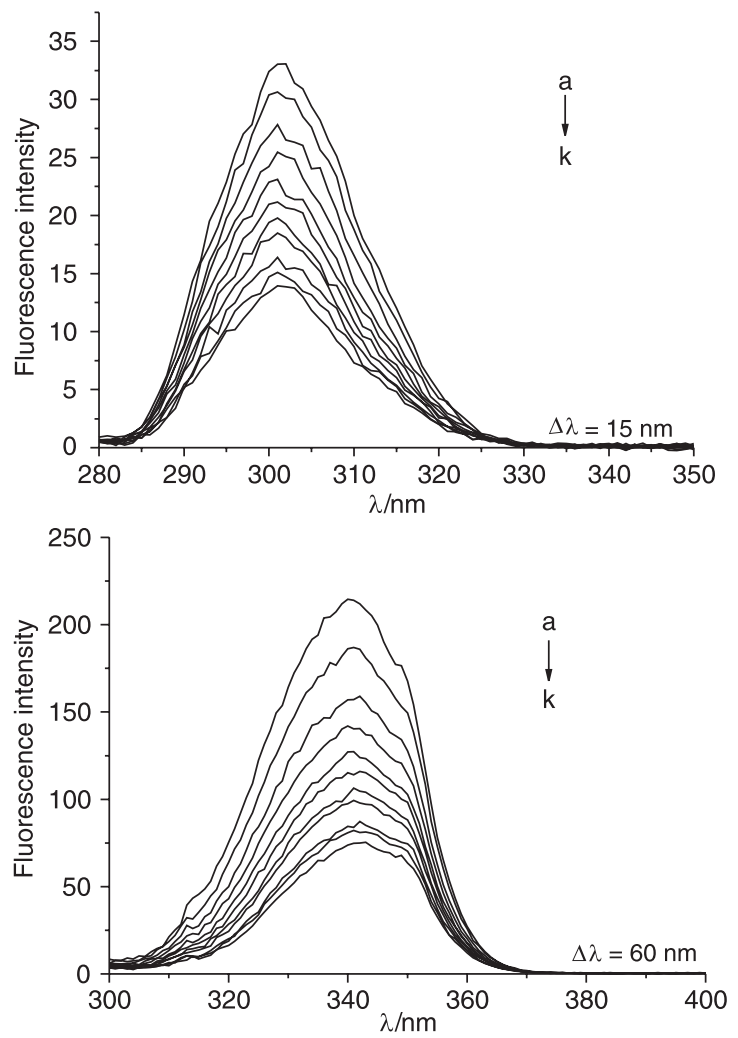

Figure 4. Synchronous fluorescence spectra of BSA upon the addition of efonidipine at $298 \mathrm{~K} . C_{\mathrm{BSA}}=10 \mu \mathrm{M} ; C_{\text {efonidipine }}$ from a to k: 0 (a), 5 (b), 10 (c), 15 (d), 20 (e), 25 (f), 30 (g), 35 (h), 40 (i), $45(\mathrm{j})$, and $50(\mathrm{k}) \mu \mathrm{M}$.

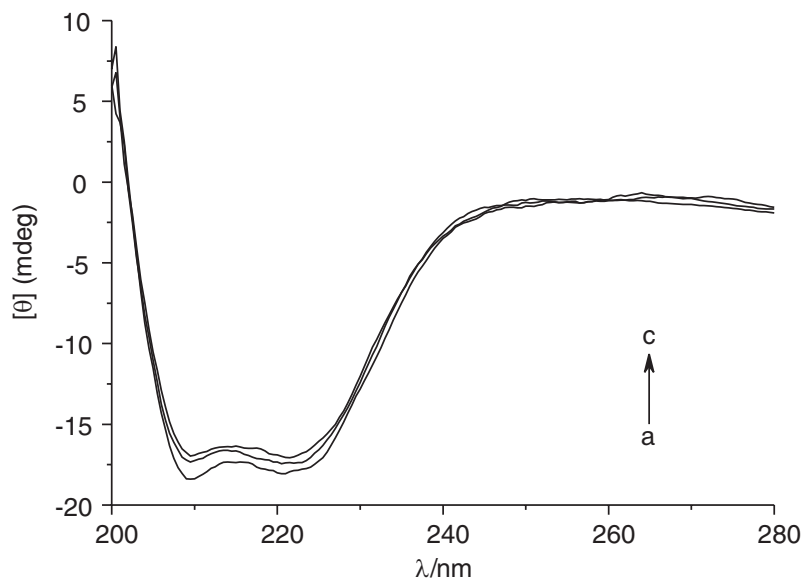

Figure 5. $C D$ spectra of $B S A$ in the presence of efonidipine. $C_{\mathrm{BSA}}=1 \mu \mathrm{M} ; C_{\text {efonidipine }}: C_{\mathrm{BSA}}$ from a to $\mathrm{c}$ are $0: 1,3: 1$, and $10: 1$. 


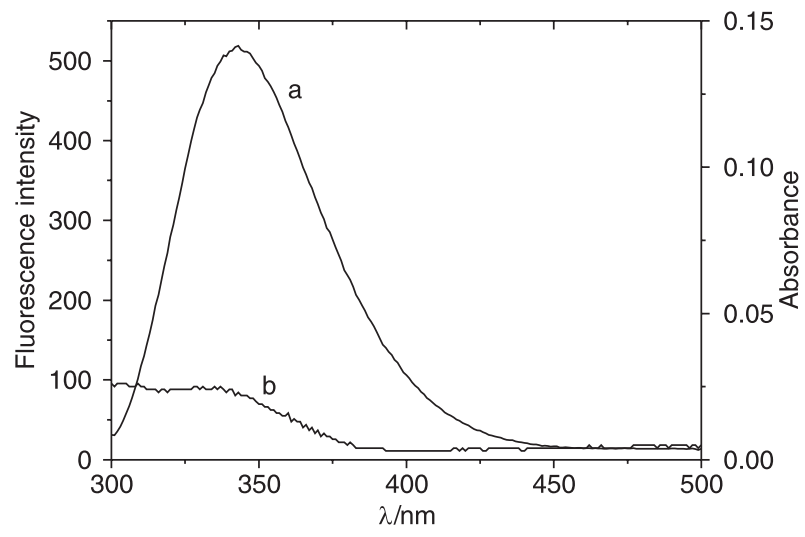

Figure 6. Spectral overlap between BSA fluorescence spectrum (a) and efonidipine UV-Vis absorbance spectrum (b). T = 298 K. $C_{\text {efonidipine }}: C_{\mathrm{BSA}}=1: 1$.

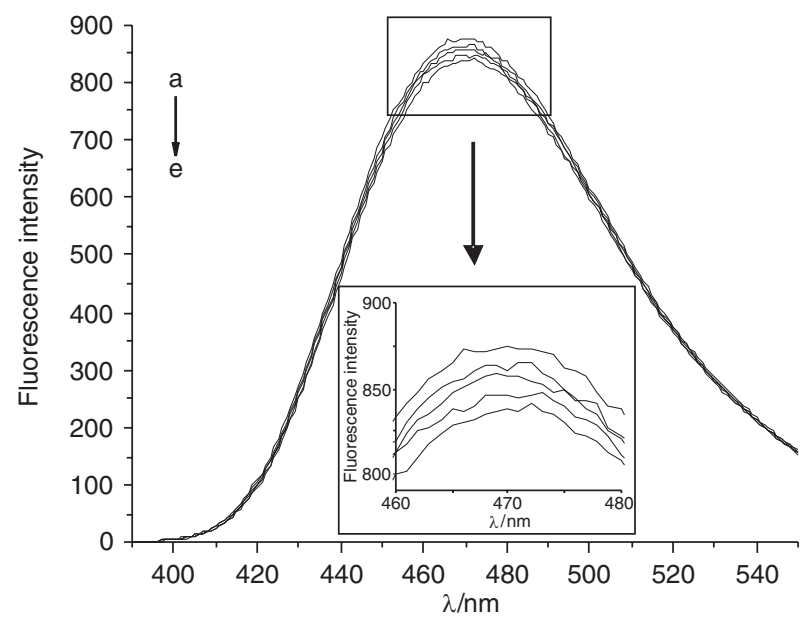

Figure 7. Fluorescence quenching spectra of ANS in the ANSBSA system at different concentrations of NZ-105 at $298 \mathrm{~K}$;

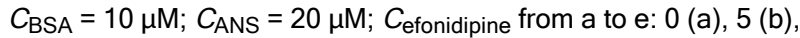
10 (c), 15 (d), and 20 (e) $\mu \mathrm{M}$.

Table 4. $K_{A}$ binding constants of the efonidipine-bovine serum albumin complex in the presence of various metal ions at $298 \mathrm{~K}$.

\begin{tabular}{lccc}
\hline System & $K_{A}\left(\times 10^{4} \mathrm{M}^{-1}\right)$ & $\mathrm{R}^{2}$ & $\mathrm{SD}$ \\
\hline BSA-efonidipine $^{2}$ & 5.58 & 0.9997 & 0.0057 \\
BSA-efonidipine-Al $^{3+}$ & 11.6 & 0.9987 & 0.0131 \\
BSA-efonidipine-Ca $^{2+}$ & 10.1 & 0.9964 & 0.0219 \\
BSA-efonidipine- $^{+}$ & 7.41 & 0.9998 & 0.0046 \\
BSA-efonidipine-Mg $^{2+}$ & 13.9 & 0.9956 & 0.0248 \\
BSA-efonidipine-Zn $^{2+}$ & 6.03 & 0.9950 & 0.0250 \\
\hline
\end{tabular}

The concentrations of bovine serum albumin (BSA), efonidipine, and metal ion were $10 \mu \mathrm{M}, 0-50 \mu \mathrm{M}$, and $10 \mu \mathrm{M}$. other for the binding sites on albumin in blood. The fluorescence studies were supplemented with the experiment of competitive binding of the fluorescent probe ANS. In the presence of NZ-05, ANS is one of the most universally used drugs in protein research. The fluorescence spectra of BSA show that both NZ-105 and ANS quench the fluorescence of BSA. The binding capacity of BSA to ANS/ NZ-105 can be evaluated by the fraction of occupied sites $(\theta)$ described as follows:

$\theta=1-\digamma / F_{0}$

(Equation 9)

where $F_{0}$ and $F$ are fluorescence intensities measured at the maximum of the emission band, in the absence and presence of ANS/NZ-105, respectively. The value of $\theta$ for the ANS-BSA system is $85 \%$, whereas for NZ-105-BSA system, it is $53 \%$. This indicates that the magnitude of decrease in fluorescence intensity is much larger for ANS than for NZ-105, which suggest that the interaction of ANS with BSA is stronger than that of NZ-105 with BSA. This may be explained by the fact that the tryptophan residues are fully accessible to the hydrophobic probe ANS, but are only partially accessible to NZ-105.

Next, the BSA-ANS interaction was studied upon gradual addition of NZ-105 by monitoring the changes in ANS fluorescence intensity upon excitation at $370 \mathrm{~nm}$. The fluorescence intensity of ANS exhibited a slight decrease upon the addition of NZ-105 (see Figure 7). Note that methanol did not affect the fluorescence intensity of ANS. The decrease observed in ANS fluorescence intensity upon the addition of NZ-105 suggests that NZ-105 displaced ANS from its binding site. Thus, NZ-105 can compete with ANS for hydrophobic sites on the surface of BSA, which means that ANS and NZ-105 do share same binding site in BSA.

\section{Influences of common ions on the binding constant}

Common ions are widely distributed in human blood and they have a definite ability to bind proteins (26), hence affecting the binding of drugs to albumin. To investigate the effect of ions on drug-albumin binding, the binding constants were determined in the presence of various ions. The values of the apparent binding constant $K_{A}$ as acquired are listed in Table 4.

As shown in Table 4, the presence of $\mathrm{Zn}^{2+}, \mathrm{Mg}^{2+}, \mathrm{Al}^{3+}$, $\mathrm{K}^{+}$, and $\mathrm{Ca}^{2+}$ increases the binding constant of the NZ-105BSA complex, indicating stronger binding of NZ-105 to $B S A$. The higher binding constants may result from the formation of metal ion-NZ-105 complexes via a metal ion bridge, which further interacts with the protein. This may 
prolong the time during which NZ-105 remains in blood and thus enhance its maximum effects (25).

The values of the thermodynamic parameters $\Delta H^{0}, \Delta S^{0}$ and $\Delta G^{0}$ at different temperatures demonstrate that the hydrophobic interaction force plays a major role in the interaction of NZ-105 with BSA. The results of synchronous fluorescence spectroscopy and $C D$ show that the conformation of BSA changes in the presence of NZ-105. The interaction of NZ-105 with BSA is weaker than that of ANS with BSA. However, NZ-105 can compete with ANS for hydrophobic sites on the surface of BSA. Furthermore, the presence of metal ions such as $\mathrm{Zn}^{2+}, \mathrm{Mg}^{2+}, \mathrm{Al}^{3+}, \mathrm{K}^{+}$, and $\mathrm{Ca}^{2+}$ increases the binding constant of the efonidipineBSA complex.

\section{References}

1. Flarakos J, Morand KL, Vouros P. High-throughput solutionbased medicinal library screening against human serum albumin. Anal Chem 2005; 77: 1345-1353.

2. Mallick A, Bera SC, Maiti S, Chattopadhyay N. Fluorometric investigation of interaction of 3-acetyl-4-oxo-6,7-dihydro$12 \mathrm{H}$ indolo-[2,3-a] quinolizine with bovine serum albumin. Biophys Chem 2004; 112: 9-14.

3. Kandagal PB, Ashoka S, Seetharamappa J, Shaikh SM, Jadegoud $Y$, ljare $O B$. Study of the interaction of an anticancer drug with human and bovine serum albumin: spectroscopic approach. J Pharm Biomed Anal 2006; 41: 393-399.

4. Sun SF, Zhou B, Hou HN, Liu Y, Xiang GY. Studies on the interaction between Oxaprozin-E and bovine serum albumin by spectroscopic methods. Int J Biol Macromol 2006; 39: $197-200$.

5. He XM, Carter DC. Atomic structure and chemistry of human serum albumin. Nature 1992; 358: 209-215.

6. Yotsumoto T, Masuda Y, Shudo C, Sugita H, Yamashita T, Tanaka S. Effects of efonidipine hydrochloride (NZ-105), a calcium antagonist, on renal function in conscious spontaneously hypertensive rats. Gen Pharmacol 1995; 26: 333337.

7. Takeda M, Shou I, Tomino Y. Effects of the antihypertensive drug efonidipine hydrochloride on albuminuria and renal histopathology in young spontaneously hypertensive rats with diabetes. Gen Pharmacol 1998; 30: 749-752.

8. Masumiya H, Shijuku T, Tanaka H, Shigenobu K. Inhibition of myocardial L- and T-type $\mathrm{Ca}^{2+}$ currents by efonidipine: possible mechanism for its chronotropic effect. Eur $J$ Pharmacol 1998; 349: 351-357.

9. Hayashi K, Kumagai H, Saruta T. Effect of efonidipine and ACE inhibitors on proteinuria in human hypertension with renal impairment. Am J Hypertens 2003; 16: 116-122.

10. Hu YJ, Liu Y, Zhang LX, Zhao RM, Qu SS. Studies of interaction between colchicine and bovine serum albumin by fluorescence quenching method. J Mol Struct 2005; 750: 174-178.

11. Wang $Y Q$, Zhang HM, Zhang GC. Studies of the interaction between palmatine hydrochloride and human serum albumin by fluorescence quenching method. J Pharm Biomed Anal 2006; 41: 1041-1046.

12. Wang CX, Yan FF, Zhang YX, Ye L. Spectroscopic investigation of the interaction between rifabutin and bovine serum albumin. J Photochem Photobiol A Chem 2007; 192: 23-28 .

13. Gao D, Tian $Y, B i S$, Chen $Y, Y u A$, Zhang $H$. Studies on the interaction of colloidal gold and serum albumins by spectral

methods. Spectrochim Acta A Mol Biomol Spectrosc 2005; 62: 1203-1208.

14. Hu YJ, Liu Y, Shen XS, Fang XY, Qu SS. Studies on the interaction between 1-hexylcarbamoyl-5-fluorouracil and bovine serum albumin. J Mol Struct 2005; 738: 143-147.

15. Papadopoulou A, Green RJ, Frazier RA. Interaction of flavonoids with bovine serum albumin: a fluorescence quenching study. J Agric Food Chem 2005; 53: 158-163.

16. Shang L, Jiang XU, Dong SJ. In vitro study on the binding of neutral red to bovine serum albumin by molecular spectroscopy. J Photochem Photobiol A Chem 2006; 184: 93-97.

17. Thipperudrappa J, Biradar DS, Lagare MT, Hanagodimath SM, Inamdar SR, Kadadevaramath JS. Fluorescence quenching of BPBD by aniline in benzene-acetonitrile mixtures. J Photochem Photobiol A Chem 2006; 177: 89-93.

18. Wang N, Ye L, Yan F, Xu R. Spectroscopic studies on the interaction of azelnidipine with bovine serum albumin. Int $J$ Pharm 2008; 351: 55-60.

19. Leckband $D$. Measuring the forces that control protein interactions. Annu Rev Biophys Biomol Struct 2000; 29: 1-26.

20. Ross PD, Subramanian S. Thermodynamics of protein association reactions: forces contributing to stability. Biochemistry 1981; 20: 3096-3102.

21. Yuan T, Weljie AM, Vogel HJ. Tryptophan fluorescence quenching by methionine and selenomethionine residues of calmodulin: orientation of peptide and protein binding. Biochemistry 1998; 37: 3187-3195.

22. Wei YL, Li JQ, Dong C, Shuang SM, Liu DS, Huie CW. Investigation of the association behaviors between biliverdin and bovine serum albumin by fluorescence spectroscopy. Talanta 2006; 70: 377-382.

23. Gao H, Wang YN, Fan YG, Ma JB. Interactions of some modified mono- and bis-beta-cyclodextrins with bovine serum albumin. Bioorg Med Chem 2006; 14: 131-137.

24. Cui FL, Fan J, Li JP, Hu ZD. Interactions between 1-benzoyl-4-p-chlorophenyl thiosemicarbazide and serum albumin: investigation by fluorescence spectroscopy. Bioorg Med Chem 2004; 12: 151-157.

25. Kandagal PB, Ashoka S, Seetharamappa J, Vani V, Shaikh SMT. Study of the interaction between doxepin and human serum albumin by spectroscopic methods. J Photochem Photobiol A Chem 2006; 179: 161-166.

26. Song $\mathrm{C}$, Liang $\mathrm{H}$. Study of binding competition between $\mathrm{Cu}$ (II) and $\mathrm{Zn}$ (II) to bovine serum albumin by fluorescence quenching. Spectrosc Spect Anal 2003; 23: 892-894. 